\title{
SENSOR DATA TRANSMISSION CONTROL USING SELECTIVE DYNAMIC COMPRESSION FOR MOBILE IOT DEVICES
}

\author{
Siwoo Byun ${ }^{1 *}$ \\ ${ }^{1 *}$ Dept. of Software, Anyang University, Anyang-shi, Kyonggi-do, South Korea \\ ${ }^{1 *}$ swbyun@anyang.ac.kr
}

Abstract - Sensor data management is a major component of the Internet of Things(IoT) environment. In this study, we provide a short overview through providing an analysis on wireless sensor networks and sensor databases. Second, we discuss concept of efficient sensor data transmission to cope with unstable sensor nodes and wireless communication failures.

To enhance the energy-efficiency and stability of data transmission in this unstable wireless sensor network environment, a new sensor-aware dynamic transmission scheme is proposed. In detail, the classification method for sensoraware transmission mode was devised and the dynamic transmission method was applied to the unstable nodes.

The proposed dynamic transmission management skills minimize battery waste and communication failures of unstable sensor nodes. Consequently, unnecessary communication costs and battery energy can be reduced, and overall energy balance can be maintained in the sensor data network.

Keywords - Sensor-based Compression, Sensor Devices, Sensor Database, Dynamic Data Transmission

\section{INTRODUCTION}

Recently, IoT(Internet of Things) sensor network has received significant attention in smart system areas. Small IoT devices can be designed with on-board calculations, wireless communications and sensor detection abilities. Recent IoT applications include energy usage monitoring and planning energy conservation in buildings, military and private surveillance, natural habitats monitoring for understanding environmental dynamics, and collecting data for learning environments. Early sensor nodes contain a small battery pack, an 8-bit processor, several megabytes RAM(Random Access Memory), several gigabytes Flash Memory, a low baud-rate radio, and sensors for detecting temperature, ambient light, and vibration [1,2].

IoT sensor network is different from the traditional wired and wireless networks since the application described above automatically operates without actual human presence. Since sensor device uses battery and wireless channel, the management efficiency of limited battery and substantial transmission energy may be a key design issue as compared to the traditional unconstrained system $[3,4]$. This means that reliability of sensor nodes and wireless communication is also an important design issue.

Sensor networks are data-centric since they are designed in terms of measured value rather than identified value such as IP address in conventional network communication.

Received: May 6, 2019

Reviewed: July 15, 2019

Accepted: August 7, 2019

* Corresponding Author 
That is, only data is important in sensor data networks. From this architectural point of view describe above, sensor network is treated as a huge global database called sensor database [5].

\section{PRELIMINARIES}

\subsection{WIRELESS SENSOR NETWORKS}

The IoT sensor network [6-8] consists of a number of sensor nodes. This sensor node has a small battery with limited power, and it has insufficient computational processing power. Therefore, if the storage space of the sensor node is exhausted, or if the battery is not replaced after it is exhausted, the sensor node is disabled. Moreover, if sufficient number of sensor nodes does not operate normally, the sensor network will not perform requested query operations.

In particular, mobile sensor nodes such as drones may be disconnected frequently. In other words, mobile sensor nodes may be disconnected from the connected wireless network due to battery failure or cell-to-cell movements. In addition, some users intentionally turn the mobile device on and off to save battery power.

Due to the nature of these mobile sensor nodes, network partitioning occurs more frequently in IoT data environments than in conventional data environments. Network partitioning causes serious data availability problems that hold the required data to be separated. This paper discusses both mobile and fixed sensor nodes, and assumes that the sensor nodes have limited communication channels and small batteries.

\subsection{SENSOR DATABASES}

The Table I below illustrates the sensor data. Sensor data consists of measured data such as temperature, humidity and light, as well as data stored in the sensor node itself. These sensor data are often used for data analysis after being collected, and the most workload of the sensor network is caused by read-only search queries.

The sensor data network is a shared-nothing platform since the sensor nodes are independent. Within these distributed platforms, sensor nodes can be easily added and search queries can be performed in parallel by the sensor nodes [9].

Table I. Example of Sensor Data

\begin{tabular}{|c|c|c|c|}
\hline Data & Minimum & Maximum & Unit \\
\hline Light & 300 & 700 & $\mathrm{~nm}$ \\
\hline Humidity & 5 & 95 & $\% \mathrm{RH}$ \\
\hline Temperature & -50 & 99.8 & ${ }^{\prime} \mathrm{C}$ \\
\hline Non-sensory data & \multicolumn{3}{|c|}{ Depends on the sensor node } \\
\hline
\end{tabular}

Recent column-based storage model $[9,10]$ is more advantageous than general storage models for storing sensor data. A column-based data storage store data in the order of columns and not in the order of rows (records) as in general data storages. Especially, it is more I/O efficient for read-only queries, such as sensor queries, because it only accesses the columns (or attributes) required for the queries.

To store data of computing devices, a wide variety of memory can be used (Fig. 1). Sensor nodes use flash memory owing to the low price, compactness, portability, and stability[5]. 


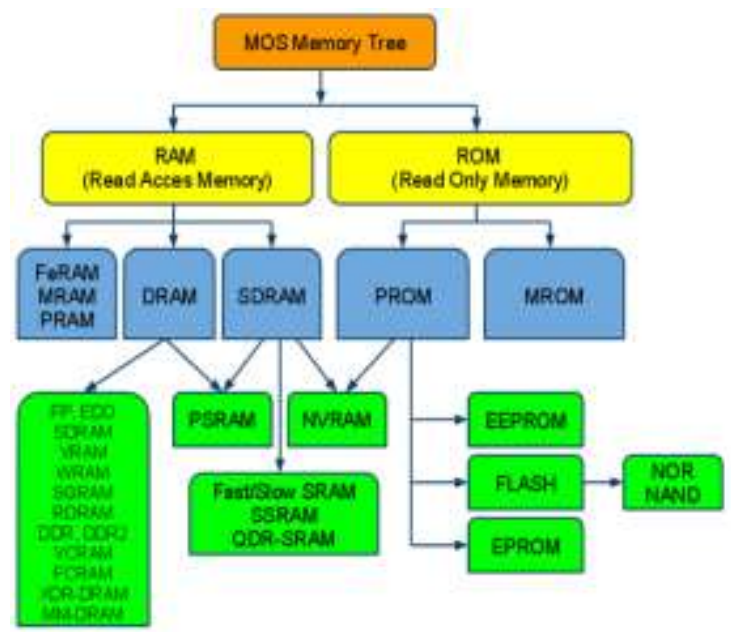

Fig. 1 Various Memory Technologies

\subsection{REDUCING ENERGY IN SENSOR NETWORK}

Some researches have been started to reduce power in the general computing environment before the sensor network was developed. Techniques such as hardware power control and database query re-balancing have been proposed to save energy on data center servers. In a typical computing environment, the CPU uses up to $60 \%$ of the whole energy at peak times, so it is important to efficiently schedule data queries to reduce power.

However, the battery energy available in the sensor network is very limited. In particular, the amount of battery consumption required for wireless communication increases significantly as transmission begins and continues to consume the battery power (Fig. 2). Therefore, the key issue to be solved is how to reduce transmission energy. For example, sensor query control study uses a node-cut strategy that dynamically generates filtering records with less computational overhead when collecting query results. This technique can reduce data comparison and calculation overhead by dynamically modifying the delivery path of sensor queries [11].

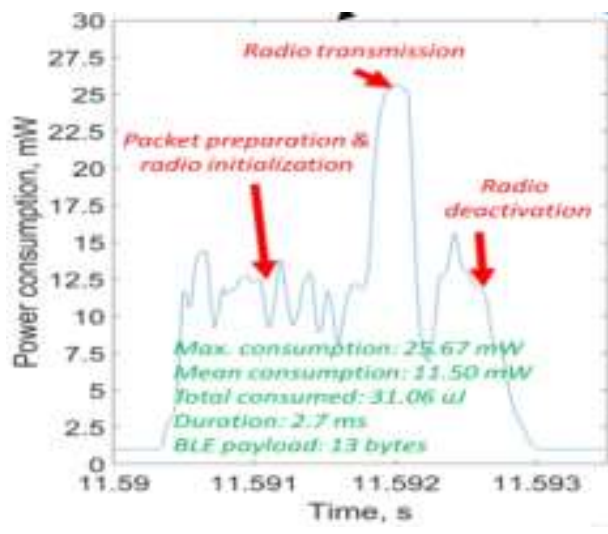

Fig. 2 Power Consumed in Packet Transmission

In addition, various studies have been conducted to increase battery life by reducing energy-consuming process in the course of data transmission in the lower layer. For example, it can reduce the number of bits to be transferred from the sensor node, or it can periodically put the sensor node into sleep mode to increase the life of the battery. It also uses energy-sensitive routing on sensor network paths to reduce energy consumption. The 
routing protocol uses energy saver and energy balancer to minimize the total energy consumed.

In summary, general transmission techniques are suitable for stable network environments, but not for unstable sensor network environments. Therefore, special consideration for energy-efficient data communication is needed for the sensor network environment.

\section{EFFICIENT TRANSMISSION CONTROL FOR SENSOR NETWORKS}

\subsection{SENSOR QUERY}

In the IoT environment, small sensor nodes have limitations such as frequently disconnected wireless channels, insufficient battery energy, and small storage capacity. In particular, the energy consumption for wireless communications is much greater than the energy consumption for sensing and computing, and the wireless network is slow to transmit at hundreds of Kbps (Table II).

Table III. Energy Consumption of Sensor Nodes

\begin{tabular}{|c|c|c|}
\hline Item & Unit & Value \\
\hline Full Battery Power & Watt(w) & 10,000 \\
\hline Average Data Rate & $\mathrm{kbps}$ & 250 \\
\hline Data Transmitting Power & $\mathrm{mW}$ & 53 \\
\hline Data Listening Power & $\mathrm{mW}$ & 57 \\
\hline Data Packet Length & Byte & $50-100$ \\
\hline Request Packet Length & Byte & $10-20$ \\
\hline
\end{tabular}

In addition, the time required to measure or access data varies for each sensor mounted on the sensor node (Table III). Therefore, these sensor specific differences are considered in the sensor query design [12].

Table IIIII. Execution Time of Sampling Operators

\begin{tabular}{|c|c|c|c|}
\hline Type & Delay(ms) & Type & Delay(ms) \\
\hline Temperature & 225 & Light & 22 \\
\hline Humidity & 78 & Non-sensory data & SN-1294 \\
\hline
\end{tabular}

In the sensor network, sensor queries use SQL-format query grammar in the form of SELECT-FROM-WHERE [9]. Sensor queries can be divided into acquisition queries and aggregation queries. In the example below, SensorQuery1 is an example of a continuous acquisition query. This query reports the sensor node ID and light intensity if the luminance reading is less than $\lambda$. SensorQuery2 is an example of an aggregation query, reporting the average temperature every 60 seconds for the sensor node in which temperature is less than $\tau[12]$.

1) SensorQuery1:

SELECT Snode_id, Snode_light FROM AllSensors WHERE Snode_light $<\lambda$

2) SensorQuery2:

SELECT AVG (Snode_temp) FROM AllSensors WHERE Snode_temp $<\tau$

With SensingInterval $=30$ 


\subsection{SENSOR-AWARE DYNAMIC TRANSMISSION SCHEME}

This study proposes stable and efficient data transmission schemes for unstable IoT sensor nodes in wireless network environments. In other words, to achieve the data transmission efficiency and stability objectives, Sensor-aware Dynamic Transmission $(\mathrm{SaDT})$ control scheme is proposed. In detail, sensor-aware classification and variable compression techniques are also presented. In case of periodic transmission, the stability and performance of the sensor database are improved by reusing last sensor data.

In order to support the stable service of the sensor database, the possibility of the sensor node failures should be considered in advance. Moreover, accumulated data generated continuously from the sensors usually has the characteristic of statistical measurements such as overall average. Therefore, if a sensor node is disabled due to battery or communication failure, the result of collective functions may be inaccurate. To cope with this problem, it is necessary to reduce battery consumption and to manage wireless communications efficiently in advance.

\subsubsection{CLASSIFICATION OF TRANSMISSION MODE}

SaDT scheme measures the service stability of each sensor node before sending data to a remote server. Based on this measurement, the proper transmission mode is determined and then the sensor data is dynamically compressed to reduce communication costs and battery power consumption.

First, the state of a sensor node is classified into two categories, depending on the stability of data transmission, in order to determine appropriate transfer mode. That is, if the battery power and the wireless signal strength are good enough, the sensor node is determined to be a normal-state. Otherwise, the sensor node is determined to be a poorstate (Fig. 3). Each sensor node maintains its time stamp for status logging when checking the remaining battery power and the radio signal strength.

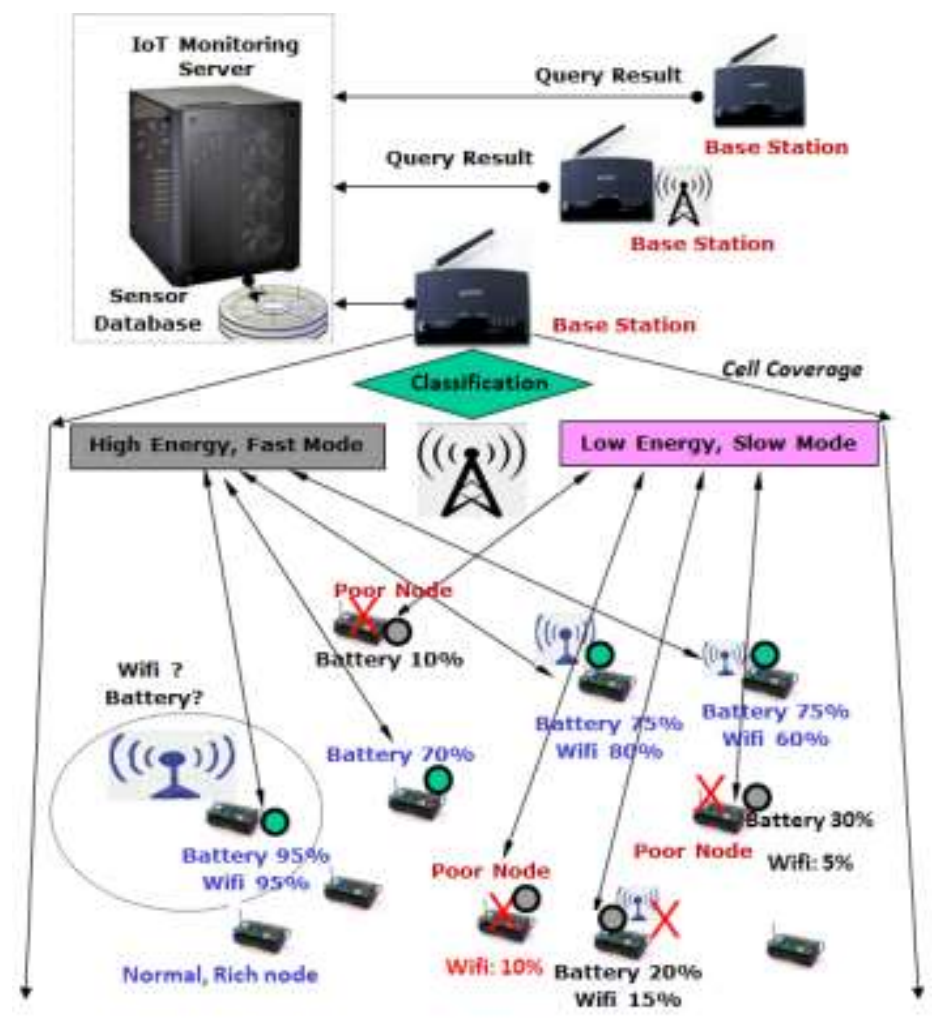

Fig. 3 State Classification of Sensor Nodes 
In $\mathrm{SaDT}$ scheme, if a sensor node is in a poor state, the immediate transmission mode is changed to be sensor-aware transmission mode which induces stable communication with low energy to successfully complete query execution. That is, SaDT reduces the amounts of incomplete communication by sending small compressed packets, and thus decreasing communication load and battery consumption.

For example, if a sensor node is under $30 \%$ of battery power and under $30 \%$ of radio signal, which is a poor state, SaDT optimizes sensor data transmission by performing selective compression and variable ratio compression.

There are two types of overhead generated by sensor nodes in poor state. One is the overhead of message re-transmissions or transmission delays due to weak radio signals. The other is a shutdown or sleep caused by lack of battery power. If this unstable or stalled node is included in the wide-area sensor query, it can lead to serious overhead in the reliability of the IoT sensor network because it causes delayed query and data loss problems during query execution.

In addition, it is efficient to send operational status of the sensor node when sending data to the base station (or gateway). The viability of each sensor node can be estimated at the level of battery charge. Generally, the voltage is expressed as a one-byte value in the range of 0 to $5 \mathrm{v}$. This status message may be sent at a certain interval.

In addition, SaDT reuse previous sensor data if the sensor data previously sent is similar to the current measured value. As a recycling effect, the effect of fast transmission and packet reduction can be achieved. Thus, overall communication cost can be reduced and overall energy efficiency can be increased. Later, when the poor sensor node becomes a normal state, it operates normally as before. Moreover, this recycling provides indirect effects such as maintaining energy balance within the sensor networks.

\subsubsection{SELECTIVE VARIABLE COMPRESSION}

The battery consumption of CPU or RAM is much lower than that of the wireless channel, and thus the battery burden of compression and decompression is relatively small. In other words, the main load of sensor nodes is the slow speed and high power consumption of wireless transmissions. The reason for compressing sensor data is that while more entries (sensor data) are stored to increase the transmission efficiency, more importantly, it can significantly reduce the power consumption required for the wireless transmission. This results in greater reliability and data availability of the entire sensor network.

In particular, in mobile sensor networks, the radio signal strength is highly variable. That is, radio signals occur at peak values in the vicinity of base stations, and the signal strength is greatly reduced as it moves away. Therefore, an effective transmission system should consider the characteristics of this highly variable radio transmission.

For example, if radio signals are very weak or battery resources are insufficient, the amount of re-transmissions is increased and the transmission speed is very slow. In this unstable case, it is more efficient to minimize the amount of data transmissions by compressing sensor data. However, if the sensor node is in normal state, it is more efficient and faster to simply send it without consuming CPU or RAM resources for compression.

However, even with sufficient batteries, if the amount of result data is increased rapidly by heavy sensor query, this also reduces transmission speed severely. Under this severe congestion, it is more efficient to make the most of the CPU resources to transmit sensor data after compression.

SaDT exploits trans-bitmap to reuse the previous sensor data. That is, if the sensor value previously sent to the base station is similar to the currently measured value within predefined range, it is reused. As a reusing effect, fast transmission and packet reduction can be achieved. That is, if the bit of a SensorNo is 1(off) in the trans-bitmap, the data 
loading is omitted, thus the data transmission can be finished quickly without consuming any subsequent compression time (Fig. 4).

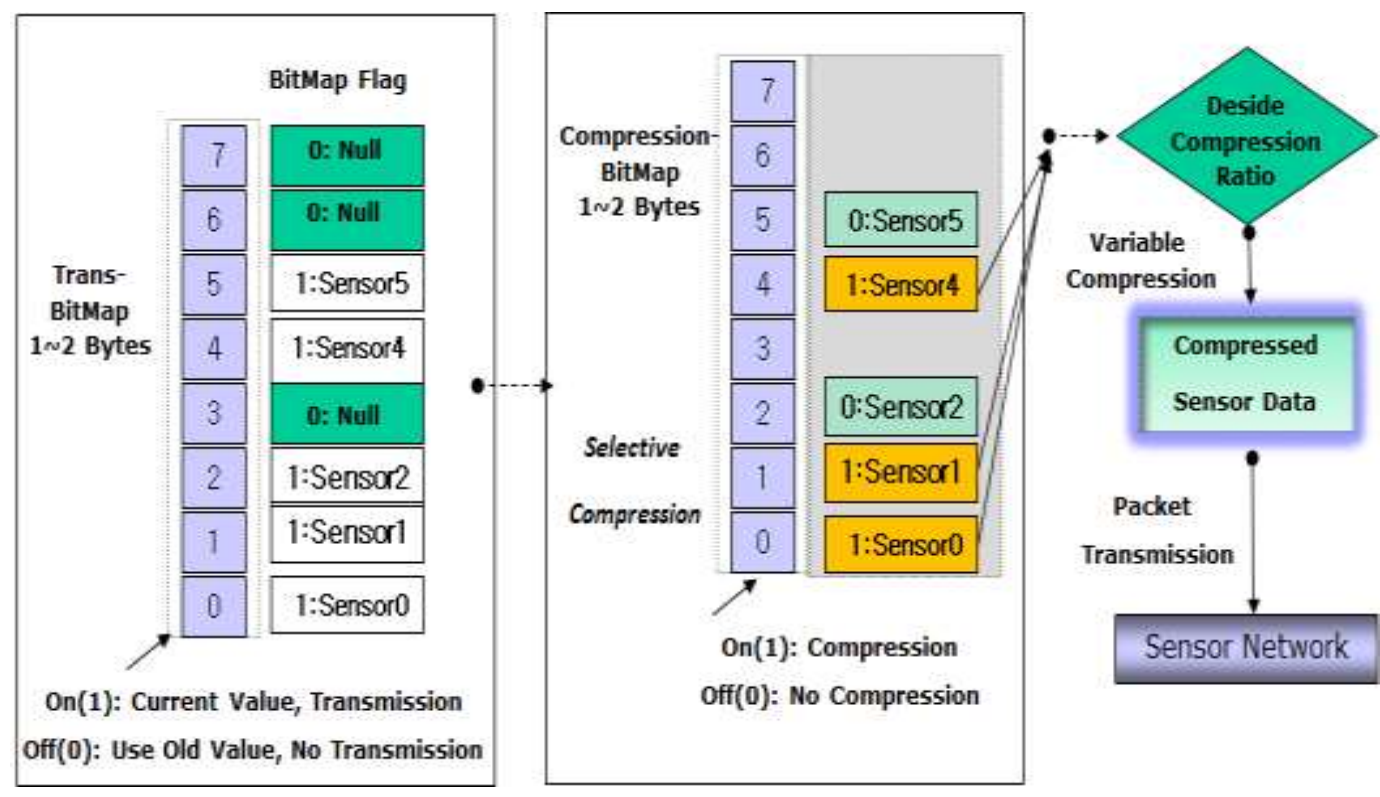

Fig. 4 Architecture of Trans-Bitmap \& Compress-Bitmap

Only in case the bit of a SensorNo is 1(on) in the trans-bitmap, SaDT determine whether to compress the sensor data or not. That is, SaDT selects only the sensor data that is well-compressed into the final transmission packets. The sensor data selected to compression is marked as 1(on) in comp-bitmap. The bit of 0(off) in comp-bitmap means that the sensor data is excluded from the next process of compression.

SaDT uses an open source Lzo-API [13] for effective compression of transmission segments. In particular, in case the amount of sensor data increases severely, SaDT exploits column-based data compression used in our previous research [14]. In this way, it is possible to reduce the amount of transmission packets and transmission power. SaDT adjusts compression efficiency and speed by varying compression level of Lzo-API. Table 4 shows the compression ratio and the compression speed according to the variable compression levels.

Table IVV. Compression Performance by Level of LZO

\begin{tabular}{|l|l|l|}
\hline $\begin{array}{l}\text { Compression } \\
\text { Level }\end{array}$ & $\begin{array}{l}\text { Compression } \\
\text { Ratio }(\%)\end{array}$ & $\begin{array}{l}\text { Compression } \\
\text { Speed (kb/s) }\end{array}$ \\
\hline Level_Low & 51.6 & 4575.3 \\
\hline Level_2 & 52.2 & 4287.3 \\
\hline Level_3 & 53.1 & 4028.1 \\
\hline$\ldots$ & $\ldots$ & $\ldots$ \\
\hline Level_9 & 54.9 & 1687.6 \\
\hline Level_High & 56.3 & 1296.7 \\
\hline Level_Optimal & 61.0 & 231.0 \\
\hline
\end{tabular}

Table 5 shows the structure of the compression segment and its sample values. Depending on the compression level, the compression speed can be adjusted by more than ten times, but three times is considered to be sufficient by default. On the other hand, 
decompression process of the sensor data is performed at high speed on a stable monitoring server with superior CPU, network bandwidth and electric power resources.

Table V. The Structure of LZO Compression for SaDT

\begin{tabular}{|l|l|l|}
\hline Segment Offset & Label & Sample Value \\
\hline$\$ 000$ & Signature & $\$ 04034 \mathrm{~B} 50$ \\
\hline$\$ 004$ & version & $\$ 0014$ \\
\hline$\$ 00 \mathrm{~A}$ & last mod. time & $\$ 11 \mathrm{AB}$ \\
\hline$\$ 00 \mathrm{E}$ & crc-32 & $\$$ \$A5F1488 \\
\hline$\$ 012$ & compressed size & $\$ 5 \mathrm{E} 3$ \\
\hline$\$ 028 \sim \$ 0 A 1$ & encoding fields & $\$ 83, \$ 68, \ldots \$ 79$ \\
\hline$\$ 0 \mathrm{~A} 2 \sim$ & Compressed Sensor Data $\ldots$ & ED98DB92E230 \\
\hline & & 0C05DFA78A7F \\
\hline & & 89102621FFF63 \\
\hline
\end{tabular}

Consequently, the proposed SaDT effectively exploits the variability of radio signals and batteries in unstable sensor network environments, and improves transmission performance and reliability through sensor-aware classification, variable compression, and selective transmission of the sensor data.

\section{Conclusion}

This study analyzed recent technological trends of wireless sensor networks and sensor databases. It also discussed efficient energy saving technology and low-power transmission technology to cope with sensor nodes and communication failures.

In order to improve the stability and energy efficiency of data transmission for unstable sensor node in the unstable wireless networks, a new sensor-aware dynamic transmission scheme was proposed. In detail, the classification method for transmission mode was devised and selective dynamic transmission was applied to the unstable nodes.

The selective transmission skill reuses previous sensor values using transmission-bit map while variable transmission skill compresses only sensor data with large compression effects using compression-bit maps. Consequently, unnecessary communication costs and battery consumption can be reduced, and overall battery energy balance can be maintained by dynamically managing sensor nodes in the clusters.

\section{ACKNOWLEDGMENTS}

This research was supported by Basic Science Research Program through the National Research Foundation of Korea (NRF) funded by the Ministry of Education (2018R1D1A1B07044418).

\section{REFERENCES}

[1] Philippe Bonnet, Johannes Gehrke, Praveen Seshadri1. "Towards Sensor Database Systems", Proceedings of the Second International Conference on Mobile Data Management, Hong Kong, January (2011): 3-14

[2] Kim Dong-Oh, Liu Lei, Shim In-Su, Kim Jeong-Joon, and Han Ki-Joon. "Spatial TinyDB: A Spatial Sensor Database System for the USN”, Int. Journal of Distributed Sensor Networks, (2013): 1-10. 
[3] Peng Hai-yun. "Energy Consumption of Wireless Sensor Network Research Problem", International Journal of Internet of Things and Big Data, 1.1 (2016): pp. 29-36.

[4] Tuvakov Jemshit, Ju-Geon Pak and Kee-Hyun Park, "A Simple Energy Efficient Routing Algorithm for the IoT Environment", International Journal of Cloud-Computing and Super-Computing, 4.2 (2017): 712.

[5] Siwoo Byun, "Design of Efficient Index Management for Column-based Big Databases", International Journal of Internet of Things and Big Data, 2.1 (2017): pp. 23-28.

[6] Jie-Won Jung, Young-Mo Kang, "An Empirical Study on the Adoption Intention of IoT Based Smart Gas Safety Shutoff Device Service”, International Journal of Internet of Things and its Applications 2.2. (2018): 7-12

[7] Ramanpreetv Kaur Deol, Jinan Fiaidhi, Sabah Mohammed, "Intruder Detection System Using Face Recognition for Home Security IoT Applications: A Python Raspberry Pi 3 Case Study”, International Journal of Security Technology for Smart Device, 5.2, (2018): 21-32.

[8] Yunsik Son, YangSun Lee, "A Study on the Interpreter for the Light-Weighted Virtual Machine on IoT Environments", International Journal of Web Science and Engineering for Smart Devices, 3.2, (2016): pp. 19-24.

[9] Kyung-Chang Kim, Choung-Seok Kim. "Parallel Processing of Sensor Network Data using ColumnOriented Databases", AASRI Procedia, 5 (2013): 2-8.

[10] Sumin Ahn, and Kyung-Chang Kim. A. "Join Technique to Improve the Performance of Star Schema Queries in Column-Oriented Databases", Journal of Korean Institute of Information Scientist and Engineers, 40.3, (2013): 209-218.

[11] Yan Wang, WeiWei, Qingxu Deng, Wei Liu, Houbing Song. “An Energy-Efficient Skyline Query for Massively Multidimensional Sensing Data”, Sensors, 16.1, (2016): 1-19.

[12] Hejun Wu, Qiong Luo, Jianjun Li. "Alexandros Labrinidis, Quality Aware Query Scheduling in Wireless Sensor Networks", Proceedings of the 6th Workshop on Data Management for Sensor Networks, in conjunction with VLDB, Lyon, France, August (2009); 1-13.

[13] http://www.oberhumer.com/products/lzo-professional/ February (2018).

[14] Siwoo Byun, "Design of Compressed Data Transmission for Mobile IoT Devices", International Journal of Energy, Information and Communications, 10.1, (2019): 21-26. 
International Journal of Control and Automation Vol. 12, No. 8 (2019) 